\title{
SERVICIOS AVANZADOS DE TELECOMUNICACIONES APLICADOS A LA ORIENTACIÓN DE JÓVENES SIN FORMACIÓN NI EXPERIENCIA LABORAL \\ ADVANCED TELECOMMUNICATIONS SERVICES APPLIED TO THE GUIDANCE OF YOUNG PEOPLE WITHOUT TRAINING AND WORK EXPERIENCE
}

Teresa Martínez Ponce ${ }^{\star}$ y Julia Ruiz Correcher ${ }^{\star *}$

Fundación Servicio Valenciano de Empleo

\section{RESUMEN}

En 1997, nuestra entidad, Fundación Servicio Valenciano de Empleo presentó un Proyecto Piloto, dentro de la convocatoria europea Leonardo, denominado SAT ORIENTACIÓN, para implantar en la Comunidad Valenciana un sistema de orientación para jóvenes sin experiencia laboral que se soportara en sistemas avanzados de comunicaciones. Este Proyecto ha resultado ganador del I Premio Educaweb de Orientación Académica y Profesional 2008 en la modalidad Institucional.

Palabras clave: Orientación, facilitador, interactividad.

\section{ABSTRACT}

In 1997, our organization, Valencian Employment Service Foundation submitted a Pilot Project, called SAT ORIENTACIÓN, within the European Programme Leonardo. The aim of the Project was to establish in the Valencian Region a guidance system supported by advanced communications systems for young people without work experience.

Key words: Guidance, faciliteitor, interactivity.

\section{Introducción}

La Fundación Servicio Valenciano de Empleo es una entidad sin ánimo de lucro, nacida en 1995 del interés común de organizaciones empresariales, sindicales y del Gobierno de la Generalitat Valenciana, para modernizar y

\footnotetext{
* Técnico de la FSVE. $t$ martinez@sve.es

** Jefa Área de Proyectos y Desarrollo de Personas FSVE. julia ruiz@sve.es
} 
mejorar la intermediación en el mercado de trabajo, mediante un ajuste eficaz de las demandas de empleo con las ofertas de puestos de trabajo de las empresas.

En la práctica surgían dificultades en esta conexión, por problemas de compatibilidad entre la Oferta y la Demanda de Empleo, ya que un porcentaje de estos demandantes, no cumplían con los requisitos que las empresas solicitaban para la cobertura de sus puestos de trabajo. Era necesario orientar a este colectivo hacia acciones que posibilitaran su inserción, con mayores garantías de éxito. La implantación de un servicio de orientación permitiría mantener un mayor contacto con estos colectivos, propiciando un asesoramiento personal y ajustado a sus necesidades.

El proyecto fué dirigido a jóvenes sin formación ni experiencia laboral y con dificultades para acceder al mercado de trabajo. También serían beneficiarios a otro nivel los profesionales de la orientación, pues el proyecto aportó un manual de formación en orientación a distancia y una herramienta de autoevaluación de orientadores. Se formó un equipo de trabajo compuesto por la Dirección del proyecto, tres técnicos para la gestión y realización de la experiencia piloto, dos personas de apoyo y una empresa externa para la coordinación del mismo. Las instalaciones utilizadas para el desarrollo del proyecto fueron nuestra sede central, sita en Valencia y las tres Agencias Territoriales de Valencia, Alicante y Castellón.

\section{Método}

El objetivo del proceso de orientación fue, guiar o facilitar la toma de decisiones de los usuarios para conseguir aumentar sus posibilidades de inserción en el mercado laboral. El colectivo objeto de intervención era el de jóvenes sin formación ni experiencia profesional.

Para la puesta en práctica de esta nueva metodología de orientación se planteó como objetivo la utilización de sistemas informáticos avanzados. Este tipo de herramienta aporta ventajas en dos sentidos:

Por un lado, plantea un servicio de orientación que no esté localizado en un punto concreto de la geografía, ya que utiliza una red de agencias interconectadas mediante una red informática donde existe un punto central que da soporte a las tareas de actualización y mantenimiento de la información, todo ello con el fin de llegar al mayor número de personas y de garantizar el uso de la misma metodología, la misma información y los mismos criterios de actuación por parte de todos los técnicos de orientación que prestan este servicio.

Por otro lado, planteó un proceso de orientación interactivo, buscando introducir el propio ordenador como elemento motivador y nexo de unión entre el técnico orientador y el usuario, con el fin de que existiera una verdadera retroalimentación entre uno y otro: la aplicación informática, por un lado recogía y por otro proporcionaba información, tanto al usuario como al orientador.

Se planteó una forma de intervención individualizada, en la que tanto el proceso en sí, compuesto por fases, como el orientador, se adecuaran a las características propias de cada usuario. Este enfoque llevaba implícita la 
voluntariedad de los usuarios ante su participación en el proceso y buscaba favorecer su implicación, ayudándoles a asumir un papel proactivo y no solo de simples receptores de información. Pretendía potenciar así su toma de decisiones, de asumir compromisos, haciéndole responsable de su propio proceso de orientación.

El propio usuario buscaba la información que necesitaba, realizaba reflexiones sobre sí mismo, tomaba decisiones, y todo ello apoyado por la figura del orientador que asumía en este caso el papel de facilitador, guía y motivador, y no sólo de conseguidor. El orientador debía ayudar a que el usuario utilizara sus propios recursos de la manera más eficaz posible, potenciándolos, motivando a ponerlos en marcha y favoreciendo un cambio de actitud hacia la búsqueda activa de empleo. La intención del orientador debía ser conseguir un compromiso de colaboración, de trabajo conjunto con el usuario, y debía transmitir desde el inicio el mensaje de que eran ellos -los usuarios, uno a uno- los que iban a conseguir trabajo, y que lograrlo, dependería de lo que hicieran y cómo lo hicieran.

Consideramos que para seguir esta metodología, esta forma de actuar motivando, informando y guiando al usuario, se debía disponer de una conexión directa con la realidad del mercado de trabajo.

En este sentido, la conexión entre orientación e intermediación constituyó una de las ventajas competitivas del proceso de orientación propuesto, ya que permitió, entre otras cosas, incluir a los usuarios, en cualquier momento del proceso, en una selección de personal para un puesto de trabajo real, si a juicio del orientador, reunía las condiciones necesarias de un candidato adecuado.

Antes de iniciar el proceso, era necesario que el usuario estuviera registrado en la base de datos de Intermediación. Para ello rellenaba previamente una ficha de recogida de datos. A partir de esta inscripción inicial comenzaba el proceso, recabando información sobre el usuario a todos los niveles, con la que poder determinar hacia dónde se le debía orientar y qué carencias tenía, para, a partir de aquí, dirigirle y orientarle hacia su incorporación a un puesto de trabajo.

El proceso de orientación contaba con ocho fases, que no seguían necesariamente un orden lineal ni eran de paso obligado (flexibilidad, según las características especificas del usuario) y que se corresponden con las áreas del software descrito posteriormente.

\section{Resultados}

\section{Software de orientación}

Este producto guía el trabajo del técnico orientador y guía al usuario en la toma de decisiones. Su objetivo es responder con calidad y de forma ágil y eficaz a las necesidades de orientación profesional. El contenido del producto consta de 8 áreas de intervención con el usuario:

- Presentación de los servicios de orientación, pasos a seguir y finalidad. 
- Conocimiento del candidato, donde se recoge información sobre el usuario, su motivación, actitud, aspectos personales.

- Definición de objetivos profesionales, donde se logra obtener una lista de profesiones acordes al perfil y preferencias del candidato. Sobre estas profesiones se indaga con el usuario sobre los conocimientos específicos y habilidades necesarias.

- Información acerca del mercado laboral. Determinadas las profesiones acordes con el usuario, se le informa sobre las tendencias del mercado relativas a esas profesiones, los requisitos más demandados.

- Redefinición de objetivos. Toma de decisiones. En esta fase de la orientación el usuario debe seleccionar un mínimo de cuatro profesiones de mayor a menor interés.

- Detección de carencias del usuario. Aquí se detectan los aspectos en los que el usuario debe mejorar para aumentar sus posibilidades de acceso al empleo. Se elabora una lista de carencias en formación, técnicas de búsqueda de empleo y habilidades sociales.

- Elaboración del proyecto personal y puesta en marcha. Se determinan las acciones que el usuario debe poner en marcha para cubrir sus carencias. Existe un compromiso del usuario.

- Seguimiento de actuaciones. Se hace un seguimiento y evaluación de las actuaciones realizadas por el usuario hasta que encuentre trabajo.

\section{Módulo de Formación de Orientadores}

Tiene por objeto responder a las necesidades de formación de los orientadores.

Los contenidos, áreas de actuación y principales aplicaciones de aprendizaje, se sostienen en mmetodologías, técnicas a utilizar, manejo de la información, capacidades y habilidades del orientador.

\section{Test de autoevaluación de orientadores}

Su objetivo es conseguir que los orientadores detecten sus necesidades de formación en su campo de actividad profesional y diseñen su propio plan de autodesarrollo. orientación:

El contenido del producto evalúa 10 aspectos claves en el proceso de

- Promoción del servicio y habilidad para implicar al usuario en el servicio de orientación.

- Desarrollo de la interacción con el usuario. Capacidad del orientador para decidir conjuntamente con el usuario.

- Detección de necesidades de información y producción de esa información.

- Evaluación del Plan de Acción. 
- Evaluación de los procedimientos de referencia que faciliten la comunicación del usuario con otras organizaciones.

- Evaluación de la colaboración en red entre organizaciones y usuarios.

- Evaluación de las relaciones del técnico orientador con los proveedores de oportunidades como centros de formación, empresas, etc.

- Evaluación de la capacidad de identificar las necesidades de aprendizaje de los usuarios respecto a sus propósitos profesionales.

- Evaluación de la capacidad de relación del orientador con sus compañeros y usuarios.

- Capacidad del orientador para autoevaluarse.

\section{Conclusiones}

La aplicación de Orientación diseñada supone una propuesta integradora, flexible y novedosa, para realizar programas de Orientación y recoger información relevante, a la vez que sirve de guia tanto al usuario como al Técnico de Orientación.

La flexibilidad del planteamiento permite darle a cada usuario lo que necesita en cada momento.

Quizas el mayor logro o innovación es la integración de esta herramienta en otra de gestión de empleo propia, de tal forma que posibilita realizar consultas sobre ofertas de empleo ON LINE, esto supone un gran paso adelante, a tenor de las impresiones recogidas en las presentaciones que realizamos a diferentes entidades.

Como síntesis de las aportaciones podemos destacar:

- Evaluación de la capacidad de relación del orientador con sus compañeros y usuarios.

- Proceso apoyado en sistemas informáticos avanzados, que permite cubrir un mayor ámbito geográfico y mantener la homogeneidad del proceso allí donde se realice.

- Flexibilidad de las acciones, permitiendo adaptar la orientación a cada usuario en particular, incidiendo en aquellos aspectos con mayores carencias en cada caso.

- Incorporación de una herramienta denominada Adult Directions que permite concretar cuales pueden ser los objetivos profesionales de un candidato, en función de sus intereses, habilidades y preferencias. Seleccionar aquellas profesiones o puestos de trabajo para los que tiene potencialidad.

- Conexión en tiempo real con el mercado de trabajo. Una vez definidos los objetivos profesionales, permite contrastar con las ofertas de empleo que se han recibido en el último periodo, pudiendo saber las ofertas que están abiertas y las que se han cerrado. Permite jugar con variables como, formación reglada, localización geográfica y puestos de trabajo 
solicitados, para obtener información de las ofertas que están abiertas con esas características o para predecir a que ofertas hubiera podido acceder si tuviera alguno de los requisitos que en la actualidad no cumple. Adicionalmente pueden consultarse los requisitos más demandados para los puestos que interesen.

- Capacidad para extraer conclusiones, elaborar informes y obtener información sobre el colectivo orientado, sobre la base de un procedimiento estadístico que permite analizar la gestión realizada sobre múltiples variables, tanto cuantitativas como cualitativas, (edad, sexo, formación, actitud ante la búsqueda de empleo, motivos para la búsqueda de empleo, carencias detectadas, ámbito geográfico, etc...).

El proyecto en toda su extensión está disponible en:

http://www.educaweb.com/esp/servicios/boletin/but081124/boletin081124.htm

Fecha de recepción: 05-02-2009

Fecha de revisión: 30-11-2009

Fecha de aceptación: 19-01-2010 